\title{
SUBPIXEL TRANSLATION OF MEMS MEASURED BY DISCRETE FOURIER TRANSFORM ANALYSIS OF CCD IMAGES
}

\author{
C. Yamahata ${ }^{1}$, E. Sarajlic ${ }^{2}$, M. Stranczl ${ }^{1}$, G.J.M. Krijnen ${ }^{3}$, and M.A.M. Gijs ${ }^{1}$ \\ ${ }^{1}$ Laboratory of Microsystems, Ecole Polytechnique Fédérale de Lausanne, Lausanne, SWITZERLAND \\ ${ }^{2}$ SmartTip B.V., Enschede, THE NETHERLANDS \\ ${ }^{3}$ Transducers Science \& Technology, MESA+, University of Twente, Enschede, THE NETHERLANDS
}

\begin{abstract}
We present a straightforward method for measuring in-plane linear displacements of microelectromechanical systems (MEMS) with subnanometer resolution. The technique is based on Fourier transform analysis of a video recorded with a Charge-Coupled Device (CCD) camera attached to an optical microscope and can be used to characterize any device featuring periodic patterns along the direction of motion. Using a digital microscope mounted on a vibration isolation table, a subpixel resolution better than 1/100 pixel could be achieved, enabling quasi-static measurements with a resolution of $0.5 \mathrm{~nm}$.
\end{abstract}

\section{KEYWORDS}

Optical measurement, subpixel resolution, discrete Fourier transform, phase-shift measurement

\section{INTRODUCTION}

For calibration and application of MEMS sensors and actuators, it is often required to measure in-plane motion of mechanical structures with a high position resolution. Optical techniques that combine digital image processing with stroboscopic video microscopy have been successfully implemented in state-of-the-art instruments for dynamic in-plane characterization of MEMS [1-4]. Here, we propose an in-plane measurement method that does not require any special investment in the measurement equipment and can be used in almost any laboratory, since its implementation only requires a CCD camera and a bright field microscope [5]. Our method is capable of observing in-plane motion with a resolution of $0.5 \mathrm{~nm}$ for a pixel size equivalent to $75 \mathrm{~nm}$ on the sample. When recording the video with a high-speed camera, dynamic characterization could be performed with a temporal resolution of $70 \mu \mathrm{s}$.

\section{WORKING PRINCIPLE}

The working principle of our method is summarized in Figure 1. First, the motion of a structure that contains periodic patterns (period $T_{\text {sens }}$ ) is recorded with a CCD camera mounted on an optical microscope. Subsequently, the image frames are cropped to the pixel area of interest (or region of interest, ROI). For each still picture (recorded at time $t$ ), a discrete intensity profile is calculated by averaging the gray value of each pixel column. The intensity profile of the first frame (time $t_{0}$ ) serves as a reference for the calculations. The displacement $\Delta x(t)$ along the $x$-axis is retrieved for each frame using a phase-shift calculation obtained from the Discrete Fourier Transform (DFT) of the intensity profile. To improve the accuracy, we have also included periodic patterns on a reference structure (period $T_{\text {ref }}$ ). The displacement of the moving structure relative to the reference is simply obtained by subtraction, thereby eliminating common noise sources. For further details on the calculation algorithm, the reader is referred to reference [5].
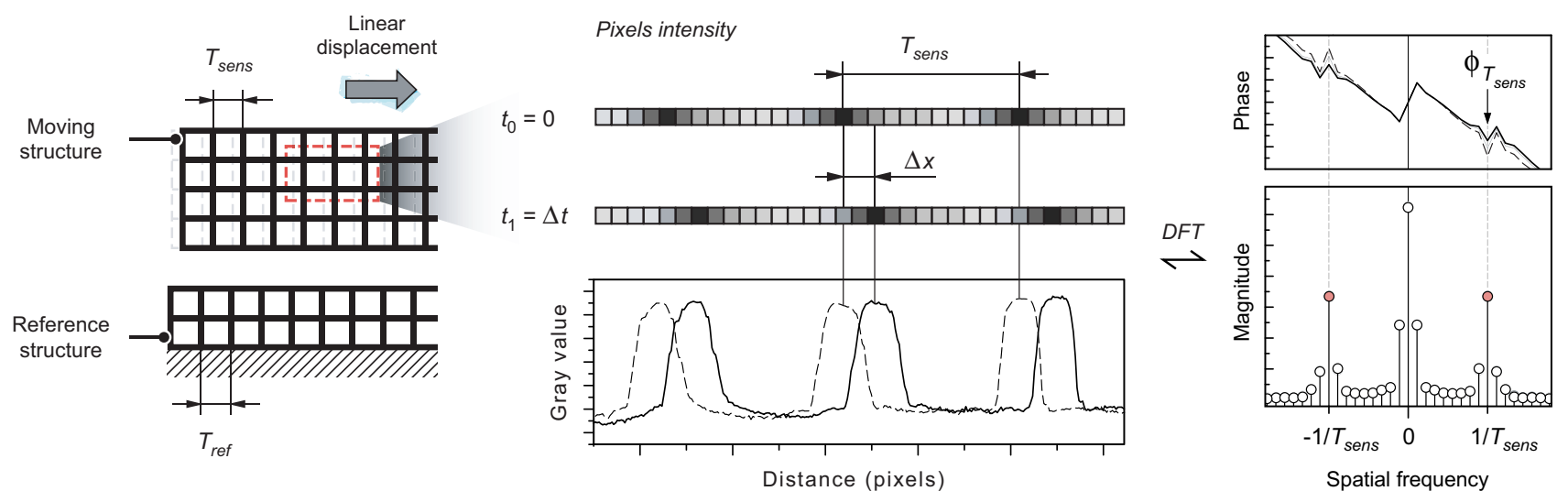

Figure 1: Principle of the measurement method. A CCD camera attached to a microscope simultaneously records periodic patterns of a reference and of a moving structure (spatial period $T_{\mathrm{ref}}$ and $T_{\mathrm{sens}}$, respectively). The motion-induced change in gray scale of pixels yields the displacement $\Delta x$, as calculated using Discrete Fourier Transform (DFT) phase-shifting. The dashed and full lines in the upper right panel correspond to the phase obtained from the pixels gray values at $t_{0}$ and $t_{1}$, respectively. The displacement $\Delta x$ is directly obtained from the phase change $\phi_{T_{\mathrm{sens}}}$ at the spatial frequency $1 / T_{\mathrm{sens}}$. 

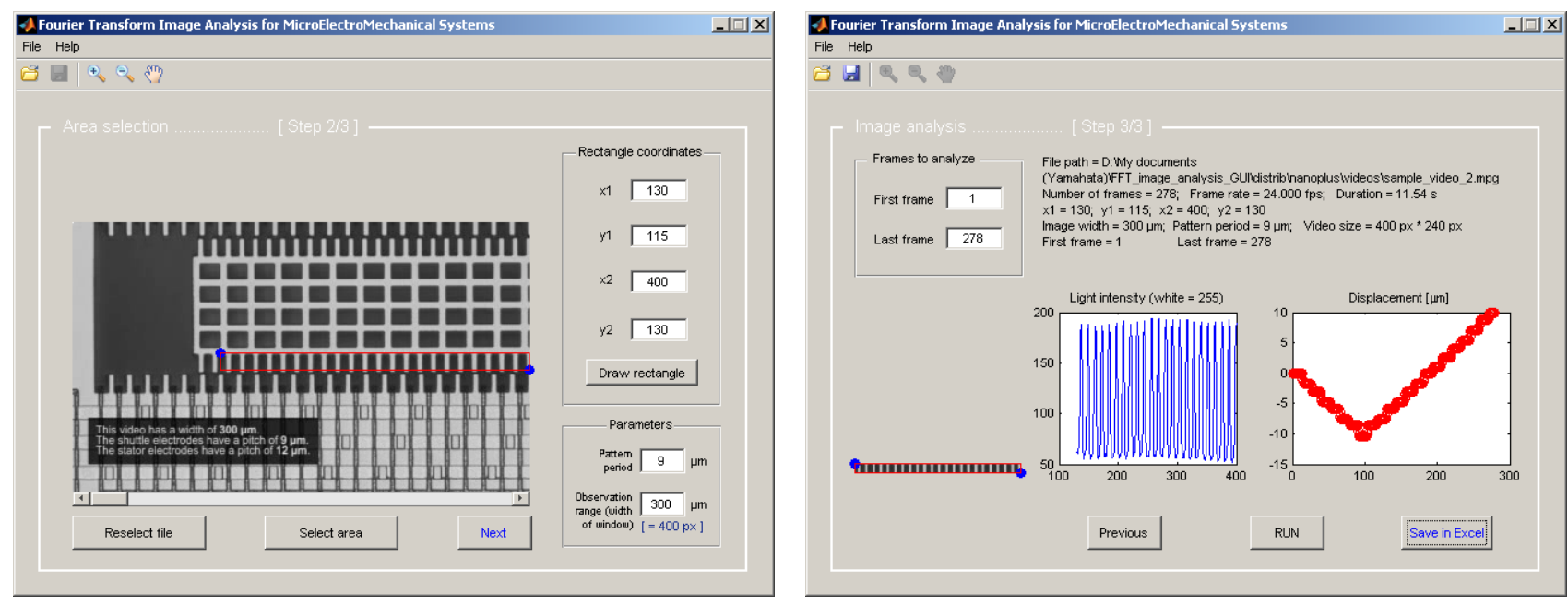

Figure 2: Screen captures of the software nano+. In this example, we have loaded a nano+ sample video (see also Figure 3). After definition of the dimensional parameters and selection of the region of interest (ROI), the DFT analysis is automatically performed frame by frame. At the end of the analysis, the displacement graph is displayed and can be saved as an Excel file.

\section{SOFTWARE DESCRIPTION}

To automate the video analysis, we have developed a MATLAB-based dedicated software (nano+). The executable file, as well as the open source code, can be downloaded from our institutional website [6]. The self-extracting executable is a standalone application based on MATLAB ${ }^{\circledR}$ and the Image Processing Toolbox $^{\mathrm{TM}}$ (The MathWorks, Inc.) that can be run without MATLAB on Microsoft Windows operating systems (software compatibility successfully tested on Windows 2000, XP, Vista and 7). In Figure 2, we show two screen captures of the Graphical User Interface (GUI). The sample video displayed in this example is that of a linear stepper micromotor fabricated by vertical trench isolation technology [7]. For video analysis, the GUI operates in three steps:

- Step \#1: Selection of the video file. It is advisable to use an uncompressed AVI file. The use of other video formats is possible provided that they are compressed with a MATLAB compatible codec. Since compression implies loss of information, the choice of the video format is a matter of trade off between measurement accuracy and size of data / speed of calculations.

- Step \#2: Selection of the ROI (in pixels) and introduction of the dimensional parameters (in micrometers). For the calculations, it is necessary to provide both the pitch of the repeating structures and the width of the video (horizontal observation range).

- Step \#3: Selection of the frame range. If only a part of the video is to be analyzed, the user can select the first and last frames of the sequence. In all cases, the calculations are performed using the first analyzed frame as a reference (zero position).

Once the analysis is complete, the results are displayed and data can be saved in a Microsoft Excel file (compatibility tested with Office 97, 2000, 2007).
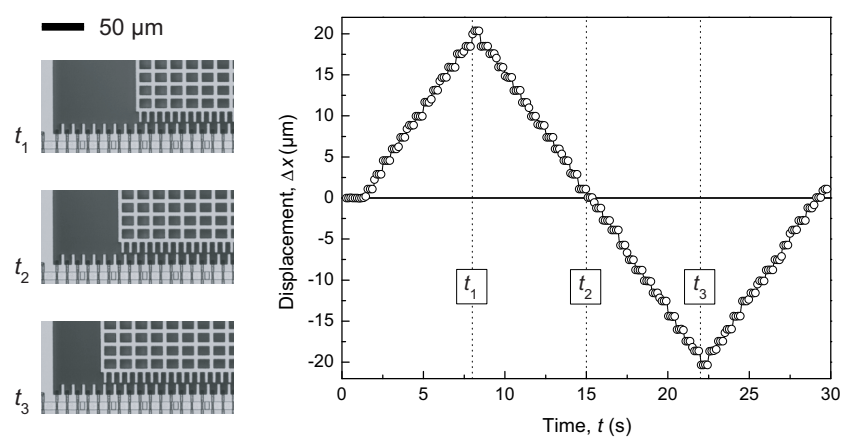

Figure 3: Example of a quasi-static displacement measurement performed by DFT analysis on a linear stepper micromotor [7]. The frame shots show the mobile shuttle of the motor in three different positions, separated by several tens of micrometers. The average displacement per step is $1.43 \mu \mathrm{m}$.

\section{EXPERIMENTAL RESULTS}

To demonstrate the potential of our method, we have used our software to analyze videos of various examples of moving silicon microdevices. All the experiments were performed on a vibration isolation table (TMC 63-534). For dynamic characterization with videos recorded at high frame rates, we have used a CMOS high-speed camera EoSens MC1363 and a MotionBLITZ-LTR1 long time recording system (Mikrotron GmbH, Germany). The other videos were recorded with a digital microscope VHX-600E, using a $\times 5000^{\text {a }}$ zoom lens VH-Z500R (Keyence Corp., Japan).

\section{Static Characterization of Various MEMS Devices}

In Figure 3, we show typical results for an electrostatic linear stepper micromotor moving back and forth (see also Figure 2) with a displacement range of

\footnotetext{
${ }^{a}$ Ratio between the apparent size on the 15 -inch LCD monitor of the digital microscope and the true size.
} 


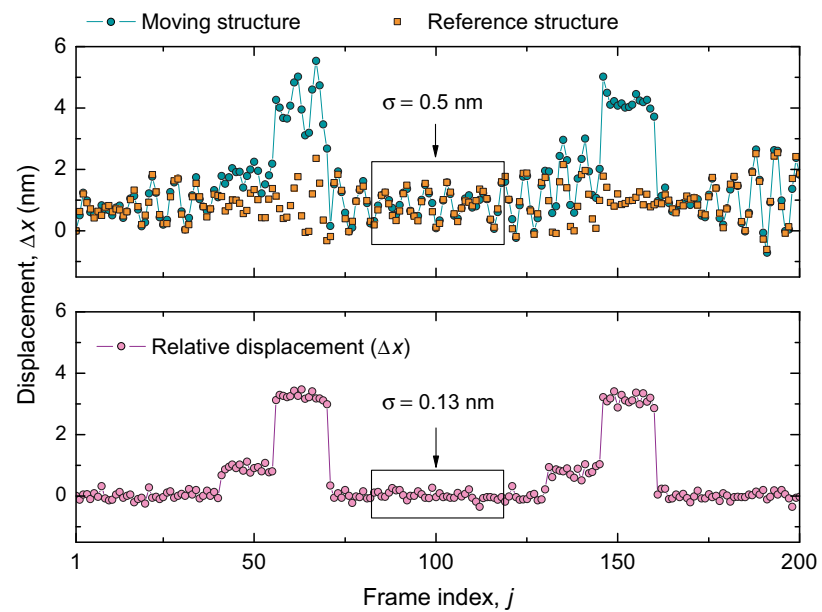

Figure 4: Subpixel resolution (better than 1/100 px) could be achieved with the DFT algorithm. In this example, a push-pull electrostatic comb-drive actuator was excited to produce steps of few nanometers. We clearly see that the reference patterns can dramatically improve the standard deviation $\sigma$ of the measurements.

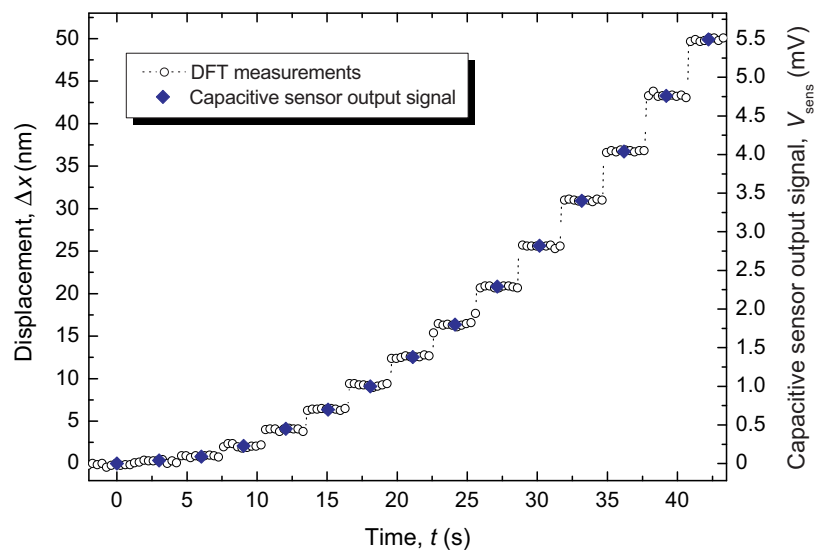

Figure 5: Output signal of a differential capacitive sensor, $V_{\text {sens, }}$ versus time upon actuation with a comb-drive, and comparison with the displacement $\Delta x$ measured by the DFT method.

several tens of micrometers [7].

In Figure 4, we show data extracted from the video of a comb-drive electrostatic actuator that was excited sequentially with very low voltages to achieve nanometer-scale displacements. We clearly see the benefit of using a reference probe to decrease the standard deviation $\sigma$ by a factor $>3$ and reach a subnanometer resolution of $4 \sigma \approx 0.5 \mathrm{~nm}$. The pixel width being $75 \mathrm{~nm}$ in this example, this performance corresponds to a subpixel resolution better than $1 / 100 \mathrm{px}$.

In Figure 5, we have recorded the output signal $V_{\text {sens }}$ of a capacitive sensor monitored using a lock-in amplifier [8] and compared these results with displacement data obtained from our DFT phase-shifting method. While the output signal of the solid-state capacitive sensor is

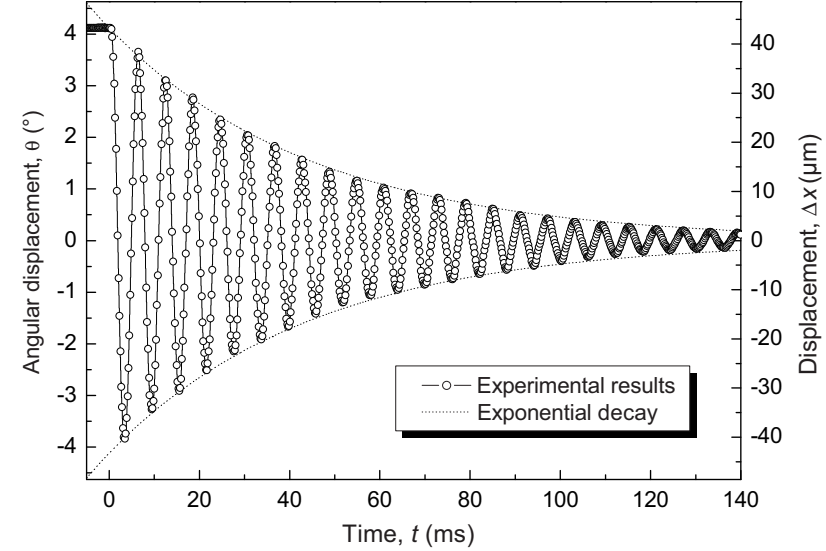

Figure 6: Transient response of a rotating microactuator [9]. The data were extracted from a video recorded with a high-speed CCD camera (frame rate of 15'000 fps). In this example, the resonant frequency is found to be $163 \mathrm{~Hz}$.

dependent on the dimensions of the etched microstructures (gap between the capacitor plates, aspect ratio), the measurements obtained with the DFT method are insensitive to process variations. Hence, this result shows a direct application of our method for the calibration of the capacitive sensor.

\section{Application to Dynamic Measurements}

Transient Response - As illustrated in Figure 6, the method is naturally extendible to dynamic measurements. Here, we have used a high-speed camera that was set to record the motion of a rotating microactuator at $15^{\prime} 000$ fps [9]. By recording the step response, our method could be used for retrieving the in-plane resonant frequency and the damping coefficient of the device. Though powerful, we do not discuss this approach further here, since it is trivial in view of the results previously exposed.

Temporally Aliased Video Microscopy - In signal sampling theory, aliasing refers to the phenomenon whereby a periodic signal is not faithfully represented in the sampled signal due to undersampling. Let's describe aliasing mathematically considering a sinusoidal signal of frequency $f_{\mathrm{M}}$. When sampled with a frequency $f_{\mathrm{s}}<2 f_{\mathrm{M}}$, the resulting samples become indistinguishable from those of another sinusoidal signal of frequency

$$
f_{\text {alias }}(N)=\left|f_{\mathrm{M}}-N f_{\mathrm{s}}\right|
$$

for any integer $N$. By default, the smallest of these frequencies would be used to reconstruct the signal, leading to an erroneous reconstruction (or aliasing artifact).

On the other hand, a priori knowledge of $f_{\mathrm{M}}$ can be exploited for perfect reconstruction of the signal [10]. An illustrative example is shown in Figure 7. For this experiment, we have actuated a push-pull electrostatic comb-drive actuator with a pure sine wave [11]. The oscillation frequency of the device was set to 


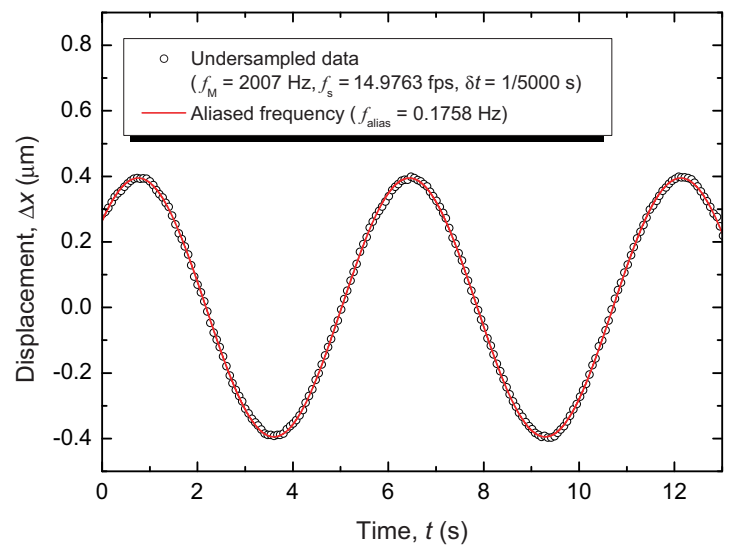

Figure 7: Principle of temporally aliased video microscopy. To illustrate temporal aliasing, the motion of a push-pull actuator oscillating at $2007 \mathrm{~Hz}$ was recorded at a frame rate of $14.9763 \mathrm{fps}$, setting the shutter aperture time to 1/5000 s. As a result, the motion is observed with an apparent frequency of $0.1758 \mathrm{~Hz}$

$f_{\mathrm{M}}=2007 \mathrm{~Hz}$. The video was recorded at a frame rate of $f_{\mathrm{s}}=14.9763 \mathrm{fps}^{\mathrm{b}}$. In order to capture unblurred images, we have used a shutter time of $\delta t=1 / 5000 \mathrm{~s}$ [12]. From (1), we find that the smallest apparent frequency is $f_{\text {alias }}=0.1758 \mathrm{~Hz}$ (for $N=134$ ). This value corresponds to the alias frequency found experimentally. The actual oscillation frequency being precisely known, this example shows that the original response could be reconstructed, opening the path for in-plane frequency response characterization.

\section{CONCLUSION AND OUTLOOK}

We have proposed a powerful approach based on digital image analysis - which can be implemented at low cost - for the in-plane static and dynamic characterization of micromechanical devices. For static measurements, we could observe in-plane motion with a subpixel resolution better than $1 / 100 \mathrm{px}$.

As discussed in the last section of this paper, the analysis of temporally aliased videos reveals further new and unexploited features of our method. We are currently developing an undersampling technique for in-plane modal analysis of MEMS.

\section{ACKNOWLEDGEMENTS}

This work was supported by the Swiss National Science Foundation (Ambizione, grant PZ00P2_121827).

\section{REFERENCES}

[1] A. Bosseboeuf, S. Petitgrand, "Characterization of the static and dynamic behaviour of $\mathrm{M}(\mathrm{O}) \mathrm{EMs}$ by optical techniques: Status and trends," $J$. Micromech. Microeng., Vol. 13, pp. S23-S33, 2003.

[2] R.M. Lin, W.J. Wang, "Structural dynamics of microsystems - Current state of research and future directions," Mech. Syst. Signal Proc., Vol. 20, pp. 1015-1043, 2006.

[3] E.M. Lawrence, C. Rembe, "MEMS characterization using new hybrid laser Doppler vibrometer / strobe video system," Proc. SPIE, Vol. 5343, pp. 45-54, 2004.

[4] Polytec Microsystem \& MEMS Analyzers products, http://www.polytec.com/int/products/microsystemmems-analyzers/.

[5] C. Yamahata, E. Sarajlic, G.J.M. Krijnen, M.A.M. Gijs, "Subnanometer translation of microelectromechanical systems measured by discrete Fourier analysis of CCD images," J. Microelectromech. Syst., Vol. 19, pp. 1273-1275, 2010.

[6] Open source code developed in MATLAB, http://lmis2.epfl.ch/nanoplus/.

[7] E. Sarajlic, C. Yamahata, M. Cordero, H. Fujita, "An electrostatic 3-phase linear stepper motor fabricated by vertical trench isolation technology," J. Micromech. Microeng., Vol. 19, no. 074001, 2009.

[8] C. Yamahata, D. Collard, B. Legrand, T. Takekawa, M. Kumemura, G. Hashiguchi, H. Fujita, "Silicon nanotweezers with subnanometer resolution for the micromanipulation of biomolecules," J. Microelectromech. Syst., Vol. 17, pp. 623-631, 2008.

[9] M. Stranczl, E. Sarajlic, G.J.M. Krijnen, H. Fujita, M.A.M. Gijs, C. Yamahata, "Modal analysis and modeling of a frictionless electrostatic rotary stepper micromotor," Proc. $24^{\text {th }}$ IEEE Int. Conf. on Micro Electro Mechanical Systems (MEMS), Cancun, Mexico, January 23-27, 2011, pp. 1257-1260.

[10] P. McCormack, "Effects and benefits of undersampling in high-speed ADC applications," Design \& Elektronik (Germany), May 2004.

[11] O. Manzardo, H.P. Herzig, C.R. marxer, N.F. de Rooij, "Miniaturized time-scanning Fourier transform spectrometer based on silicon technology," Optics Letters, Vol. 24, pp. 1705-1707, 1999.

[12] C. Rembe, R. Kant, R.S. Muller, "Optical measurement methods to study dynamic behavior in MEMS," Proc. SPIE, Vol. 4400, pp. 127-137, 2001.

\section{CONTACT}

* C. Yamahata; christophe.yamahata@a3.epfl.ch; http://christophe.yamahata.fr/

\footnotetext{
${ }^{b}$ The Keyence Digital Microscope VHX-600 is primarily aimed for high definition imaging. The recording frame rate indicated to be of 15 frames/seconds according to the technical specifications - was found to be $14.9763 \mathrm{fps}$ for our equipment.
} 